\title{
Terminology: nomenclature of mucosa-associated lymphoid tissue
}

\author{
P Brandtzaeg ${ }^{1}$, H Kiyono ${ }^{2}$, R Pabst ${ }^{3}$ and MW Russell ${ }^{4}$
}

\begin{abstract}
Stimulation of mucosal immunity has great potential in vaccinology and immunotherapy. However, the mucosal immune system is more complex than the systemic counterpart, both in terms of anatomy (inductive and effector tissues) and effectors (cells and molecules). Therefore, immunologists entering this field need a precise terminology as a crucial means of communication. Abbreviations for mucosal immune-function molecules related to the secretory immunoglobulin A system were defined by the Society for Mucosal Immunolgy Nomenclature Committee in 1997, and are briefly recapitulated in this article. In addition, we recommend and justify standard nomenclature and abbreviations for discrete mucosal immune-cell compartments, belonging to, and beyond, mucosa-associated lymphoid tissue.
\end{abstract}

\section{INTRODUCTION}

It is instructive to categorize various tissue compartments involved in mucosal immunity according to their main function. However, until recently, there was no consensus in the scientific community as to how these compartments should be named and classified. This lack of standardized terminology has been particularly confusing for newcomers to the mucosal immunology field. To address this issue, the Nomenclature Committee of the Society for Mucosal Immunology, under its chair Michael W. Russell, recommended adoption of a standard nomenclature initially proposed by Brandtzaeg and Pabst in 2004. ${ }^{1}$ This terminology was unanimously approved by the Society for Mucosal Immunology's General Assembly at the 13th ICMI in Tokyo, 2007.

This paper delineates this recommended nomenclature, with the primary aim of improving communication about the anatomy of the mucosal immune system. Before addressing this recommendation, however, we would like to recapitulate the previously proposed nomenclature for molecules related to the secretory immunoglobulin A (SIgA or S-IgA) system. This seems to be necessary as the proper terminology is often neglected by many immunologists-and even by many major journals of immunology.

\section{RECOMMENDED NOMENCLATURE FOR SECRETORY IMMUNE-FUNCTION MOLECULES}

The molecular specialization of the SIgA system was described as early as in $1965,{ }^{2}$ and a common epithelial transport model for dimers (and larger polymers) of IgA and pentamers of IgM was proposed in 1974..$^{3,4}$ The epithelial glycoprotein designated secretory component (SC) by WHO in 1972 (previously called "transport piece" or "secretory piece") turned out to be responsible for the receptor-mediated transcytosis of J-chain-containing Ig polymers (pIgs) through secretory epithelia, ${ }^{5}$ and the functional name polymeric Ig receptor (pIgR) is now commonly used for the transmembrane form of SC. During export of pIgs, the cleaved ectodomain of the pIgR is incorporated into SIgA and secretory $\operatorname{IgM}$ as the so-called bound SC, which stabilizes particularly SIgA by covalent bonding, or is released from unoccupied receptor into secretions as free SC. Bound and free SC can be distinguished by conformational epitopes ${ }^{6}$ and may in different ways exhibit innate immune functions. ${ }^{7} \mathrm{SC}$ thus exists in three forms with distinct functional roles.

The terminology of the SIg system (Table 1) was approved by the Society for Mucosal Immunology in 1997 and also by the IUIS/WHO Nomenclature Committee. ${ }^{8}$ The awareness and proper use of this nomenclature will be important for eliminating confusion when discussing this system, examples of which are the still common use of a small "s" (which stands for "surface") as prefix for the SIgs, and the often neglected specification of $\mathrm{SC}$ as being either bound or free in distinction from membrane SC.

\footnotetext{
${ }^{1}$ Laboratory for Immunohistochemistry and Immunopathology (LIIPAT), Institute and Department of Pathology, Rikshospitalet University Hospital, Oslo, Norway. ${ }^{2}$ Division of Mucosal Immunology, Department of Microbiology and Immunology, The Institute of Medical Science, University of Tokyo, Tokyo, Japan. ${ }^{3}$ Department of Functional and Applied Anatomy, Medical School of Hannover, Hannover, Germany. ${ }^{4}$ Department of Microbiology and Immunology, Witebsky Center for Microbial Pathogenesis and Immunology, State University of New York, Buffalo, New York, USA Correspondence: P Brandtzaeg (per.brandtzaeg@medisin.uio.no) 
Table 1 Recommended nomenclature for secretory immunefunction molecules ${ }^{a}$

\begin{tabular}{ll}
\hline Preferred abbreviations & Explanations \\
\hline SIgA (or S-IgA) & Secretory IgA \\
SIgM (or S-IgM) & Secretory IgM \\
plgA & Polymeric IgA \\
& Refers mainly to dimers but also \\
& includes larger polymers of J- \\
& chain-containing IgA \\
& Joining chain \\
J chain & Secretory component \\
SC & Exists in three forms: membrane \\
& SC; bound SC; and free SC \\
& Polymeric Ig receptor \\
plgR & The same as membrane SC \\
\hline
\end{tabular}

plgA, IgA dimers/polymers; plgR, polymeric Ig receptor; SC, secretory component; SlgA, secretory immunoglobulin A; SlgM, secretory immunoglobulin M. aApproved by IUIS/WHO Subcommittee on IgA nomenclature. ${ }^{8}$

\section{RECOMMENDED NOMENCLATURE FOR MUCOSA- ASSOCIATED IMMUNE-CELL COMPARTMENTS Inductive and effector tissues}

No attempt has been made previously to standardize the terminology applicable to the various tissue compartments involved in the mucosal immune system. These compartments can principally be divided into inductive sites-where antigens sampled from mucosal surfaces stimulate cognate naive $\mathrm{T}$ and B lymphocytes-and effector sites-where the effector cells after extravasation, retention, and differentiation perform their action, for instance by contributing to the formation of SIgA antibodies (Figures $\mathbf{1}$ and 2).

The inductive sites for mucosal immunity are constituted by organized mucosa-associated lymphoid tissue (MALT) as well as local/regional mucosa-draining lymph nodes (LNs), whereas the effector sites consist of distinctly different histological compartments-including the lamina propria (LP) of various mucosae, the stroma of exocrine glands, and surface epithelia. ${ }^{1}$ Peyer's patches (PPs) in the small intestine of humans, rodents, and rabbits are typical MALT structures believed to be a main

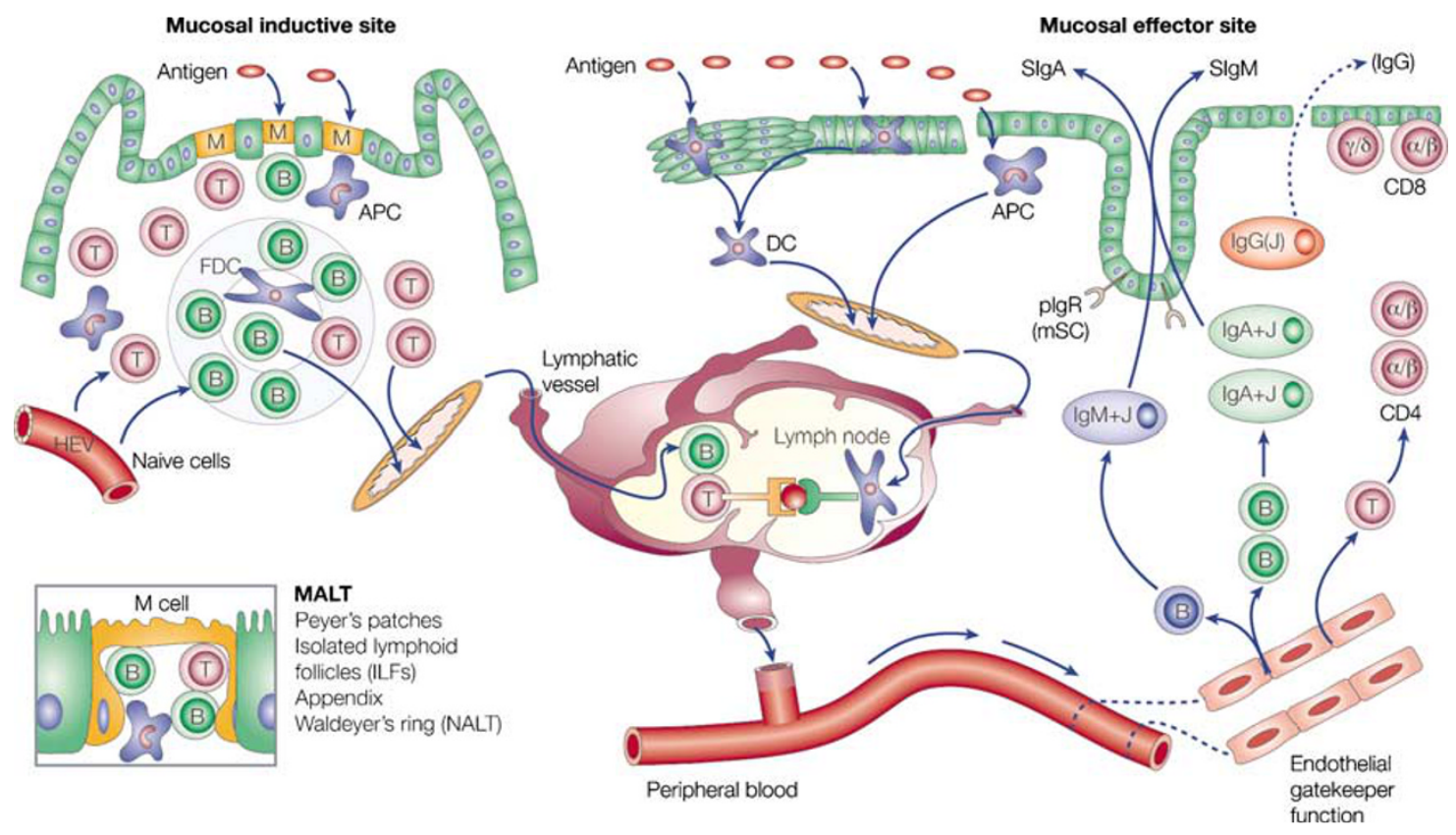

Figure 1 Depiction of the human mucosal immune system. Inductive sites for mucosal immunity are constituted by regional MALT with their B-cell follicles and M-cell (M)-containing follicle-associated epithelium through which exogenous antigens are transported actively to reach APCs, including DCs, macrophages, B cells, and FDCs. In addition, quiescent intra- or subepithelial DCs may capture antigens at the effector site (exemplified by nasal mucosa in the middle) and migrate via draining lymphatics to local/regional lymph nodes where they become active APCs, which stimulate T cells for productive or downregulatory (suppressive) immune responses. Naive B and T cells enter MALT (and lymph nodes) via HEVs. After being primed to become memory/effector B and T cells, they migrate from MALT and lymph nodes to peripheral blood for subsequent extravasation at mucosal effector sites (exemplified by gut mucosa on the right). This process is directed by the local profile of vascular adhesion molecules and chemokines, the endothelial cells thus exerting a local gatekeeper function for mucosal immunity. The gut lamina propria contains few B lymphocytes but many J-chainexpressing IgA (dimers/polymers) and IgM (pentamers) plasmablasts and plasma cells. Also, there are normally some rare IgG plasma cells with a variable $\mathrm{J}$-chain level $(\mathrm{J})$, and many T cells (mainly $\mathrm{CD} 4^{+}$). Additional features are the generation of SIgA and SlgM via plgR (mSC)-mediated epithelial transport, as well as paracellular leakage of smaller amounts (broken arrow) of both locally produced and plasma-derived lgG antibodies into the lumen. There may also be some active transport of IgG mediated by the neonatal Fc receptor (not indicated). Note that IgG cannot interact with $\mathrm{J}$ chain to form a binding site for plgR. The distribution of intraepithelial lymphocytes (mainly T-cell receptor $\alpha / \beta^{+} \mathrm{CD}^{+}$and some $\gamma / \delta^{+} \mathrm{T}$ cells) is also depicted. The inset (lower left corner) shows details of an M cell and its "pocket" containing various cell types. The cartoon is modified from Brandtzaeg and Pabst ${ }^{1}$ with permission from Elsevier. APCs, antigen-presenting cells; DCs, dendritic cells; FDCs, follicular dendritic cells; HEVs, high endothelial venules; MALT, mucosa-associated lymphoid tissue; mSC, membrane secretory component; plgR, polymeric Ig receptor; SlgA, secretory IgA; SlgM, secretory IgM. 

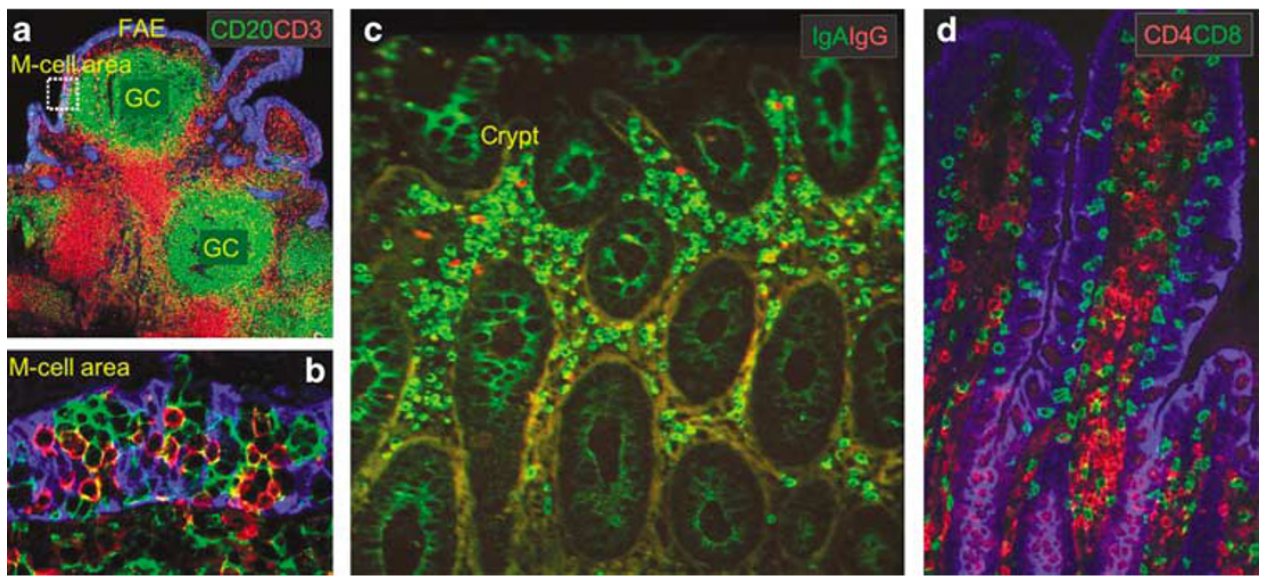

Figure 2 Illustration of three mucosal immune-cell compartments. (a,b) Three-color immunofluorescence staining of B cells (CD20, green), T cells (CD3, red), and epithelium (cytokeratin, blue) in cryosection of human Peyer's patch. (b) Details from the M-cell areas framed on the left in the follicle-associated epithelium (a) covering a B-cell follicle. (c) Two-color immunofluorescence staining for lgA (green) and IgG (red) in a section from normal human large bowel mucosa. Crypt epithelium shows selective transport of IgA, and only a few scattered IgG-producing cells are seen in the lamina propria together with numerous IgA plasma cells. (d) Three-color immunofluorescence staining for $\mathrm{CD}^{+}$(red) and CD8 ${ }^{+}$(green) T cells in normal human duodenal mucosa. The epithelium of the villi is blue (cytokeratin). Note that most of the elements with weak CD4 expression seen in the background are either macrophages or dendritic cells. The immunofluorescence pictures are original images from the Brandtzaeg laboratory, reproduced from Brandtzaeg and Pabst ${ }^{1}$ with permission from Elsevier.

source of conventional (B2) surface (s)IgA-expressing primed (memory/effector) and class-switched mucosal B cells.9,10

The term MALT was first coined to emphasize that solitary organized mucosa-associated B-cell follicles and larger lymphoid aggregates have common features and are the origin of cells that traffic to mucosal effector sites. ${ }^{11}$ MALT is sub-divided according to anatomical regions (Table 2), and the distribution and composition of such lymphoid structures vary considerably with species (Figure 3). Also the age and tissue state (normal or chronically inflamed) have an impact on the appearance of MALT. In contrast to PPs and tonsils, other human MALT structures do not apparently develop prenatally, ${ }^{1}$ and their occurrence and size generally depend on induction by exogenous stimuli. For instance, bronchus-associated lymphoid tissue (BALT) is not regularly found in normal lungs of adultsreportedly being absent also in $60 \%$ of healthy adolescents and children. ${ }^{12}$

It should be noted that although LP is considered an effector site, it is still important for the expansion of B cells and their terminal differentiation to plasma cells (PCs). ${ }^{9}$ In addition, T-cell-independent switch of B cells to IgA expression has been reported to occur in murine LP. ${ }^{10}$ This event may particularly involve the large contingent of $\mathrm{B} 1$ cells derived from the peritoneal cavity in mice-a possibility that remains controversial, however, because of discrepant findings. ${ }^{1,13}$ No evidence exists to suggest that $\mathrm{B} 1$ cells populate significantly human gut mucosa, $, 1,9$ and no convincing signs of class switch to IgA have previously been found in normal human intestinal LP. ${ }^{14}$ Nevertheless, in the large bowel, bacteria could induce switch factors in epithelial cells and LP dendritic cells (DCs) that may drive T-cell-independent B-cell development toward the IgA2 subclass. ${ }^{15}$ The first evidence for such a role of the gut microbiota was obtained as early as in 1995 by showing that jejunal segments with bacterial overgrowth had an $\sim 50 \%$ reduction of the IgA1:IgA2 mucosal PC ratio. ${ }^{16}$

Finally, expansion of memory/effector T cells can apparently take place within the surface epithelium, which is often referred to as the intraepithelial lymphocyte (IEL) compartment (Table 2). There is considerable "cross talk" both within and between the LP and IEL compartments. ${ }^{17}$

\section{The MALT concept}

The concept of MALT was originally based on mouse repopulation experiments with B cells obtained from BALT and PPs as well as the surrounding LP. ${ }^{11}$ Notably, all MALT structures resemble LNs-with variable T-cell zones intervening between the B-cell follicles-and contain a variety of antigen-presenting cells, including DCs and macrophages. However, MALT lacks afferent lymphatics because all such lymphoid structures actively sample exogenous antigens directly from the mucosal surfaces through a characteristic follicle-associated epithelium containing "microfold" or "membrane" (M) cells. ${ }^{1}$ These specialized thin epithelial cells effectively transfer soluble and especially particulate antigens such as microorganisms from the gut lumen. ${ }^{18}$

Gut-associated lymphoid tissue (GALT) comprises PPs, the appendix, and isolated lymphoid follicles (ILFs), which are considered inductive sites for mucosal $\mathrm{B}$ and $\mathrm{T}$ cells. The occurrence of other GALT-like elements such as lymphocyte-filled villi and cryptopatches is species-dependent (Figure 3), and these structures do not appear to be involved in B-cell induction. ${ }^{1}$ In fact, no conclusive immunological function has been identified for the extremely rare T-cell-dominated human lymphocyte-filled villi; ${ }^{19}$ and although cryptopatches were originally thought to be the origin of IELs in mice, ${ }^{20}$ more recent data have questioned this notion and provided evidence to suggest that IELs are thymusdependent even in this species. ${ }^{21}$ These small lymphoid structures may instead, represent precursons of marine ILFs. ${ }^{22}$ 
Table 2 Recommended nomenclature for mucosa-associated immune-cell compartments

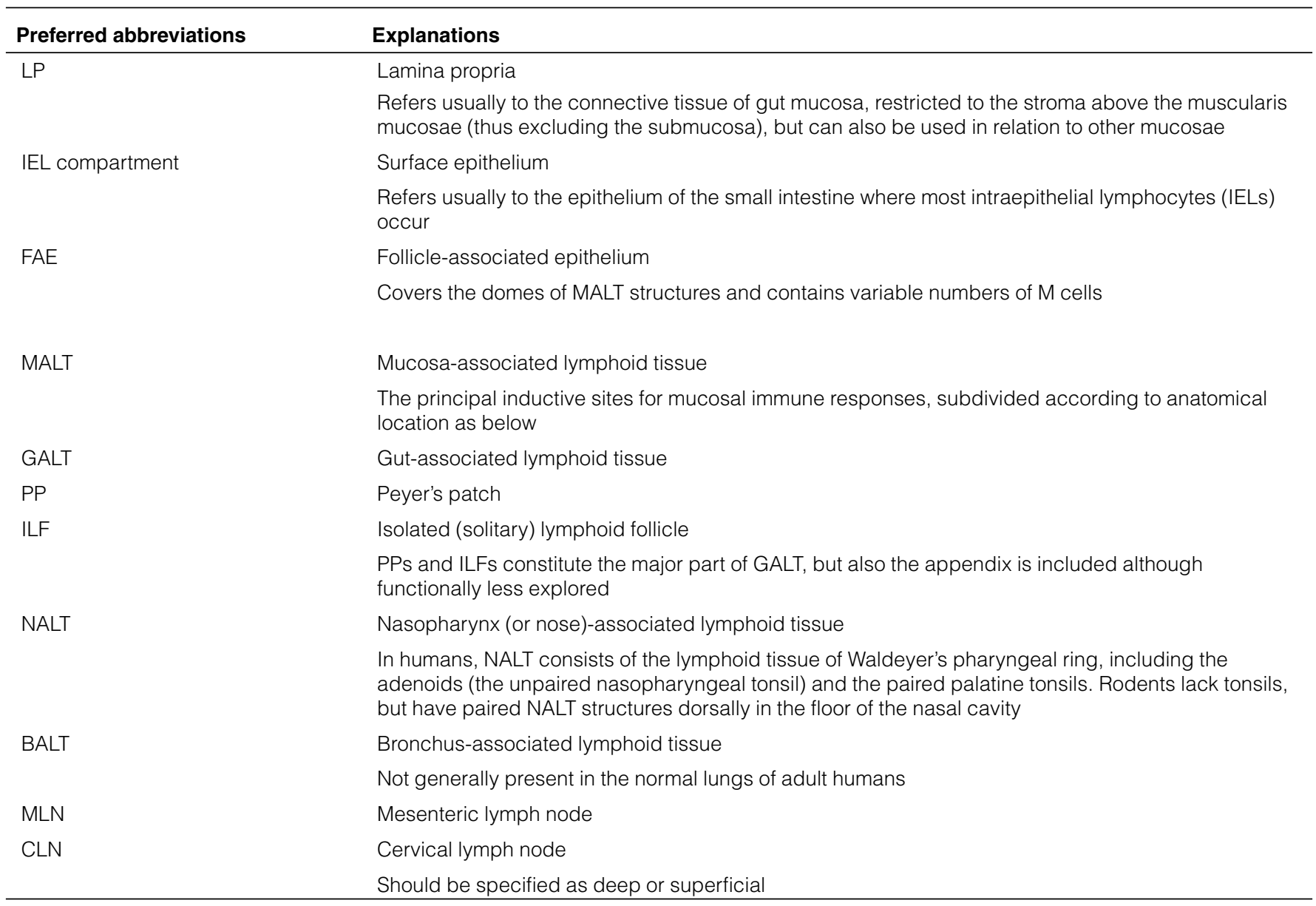

More details can be found in ref. 1

Early animal studies demonstrated that not only PPs, but also mesenteric LNs (MLNs) are enriched precursor sources for intestinal IgA-producing PCs. ${ }^{23,24}$ Differentiation of sIgA ${ }^{+} \mathrm{B}$ cells takes place during their dispersion to the effector sites. ${ }^{25,26}$ Thus, the differentiated fraction of B cells with cytoplasmic IgA was only $2 \%$ in PPs but increased to $50 \%$ in MLNs, $75 \%$ in thoracic duct lymph, and finally $90 \%$ in the intestinal LP because of terminal maturation to PCs. ${ }^{27}$ Such seminal findings gave rise to the term "IgA cell cycle." 28 In humans, a clonal relationship between sIgA ${ }^{+}$ $\mathrm{B}$ cells in PPs and IgA-producing PCs in LP provides strong support for this notion. ${ }^{29}$ Later studies have demonstrated that $\mathrm{T}$ cells as well as B cells expressing other isotypes than IgA, but induced in PPs, also exhibit gut-seeking properties. ${ }^{9}$

\section{Distribution of GALT structures}

Human PPs occur mainly in the distal ileum and contain by definition between 5 and 200 aggregated lymphoid follicles. ${ }^{30}$ Human PP anlagen, composed of $\mathrm{CD} 4^{+}$DCs, can be seen at 11 weeks of gestation, and discrete T- and B-cell areas occur at 19 weeks. However, no germinal centers appear until shortly after birth, reflecting dependency on antigenic stimulation which in childhood also induces some follicular hyperplasia, facilitating the macroscopic visibility of PPs. ${ }^{31}$ Thus, the number of macroscopically identifiable human PPs increases from some 50 at the beginning of the last trimester to 100 at birth and 250 in the midteens, then diminishes to become approximately 100 between 70 and 95 years of age. ${ }^{30}$

In addition, the human gut harbors at least 30,000 ILFs, increasing in density distally. ${ }^{32}$ The normal small intestine contains on average only 1 follicle per 269 villi in the jejunum but one per 28 villi in the ileum; ${ }^{19}$ in the normal large bowel, the density of ILFs as seen in tissue sections increases from 0.02 per $\mathrm{mm}$ length unit of muscularis mucosae in the ascending colon to 0.06 per $\mathrm{mm}$ in the rectosigmoid. ${ }^{33}$

Small-intestinal ILFs have best been characterized immunologically in mice, showing functional features compatible with induction of local IgA responses. ${ }^{34}$ Colonic ILFs are morphologically more heterogeneous, sometimes penetrating the muscularis mucosae, especially in the presence of inflammation..$^{35}$ In humans, such irregular "basal lymphoid aggregates" are much more frequent in ulcerative colitis and Crohn's disease than in the healthy large bowel, but they probably have the same functional nature as normal colonic ILFs. ${ }^{36}$ 


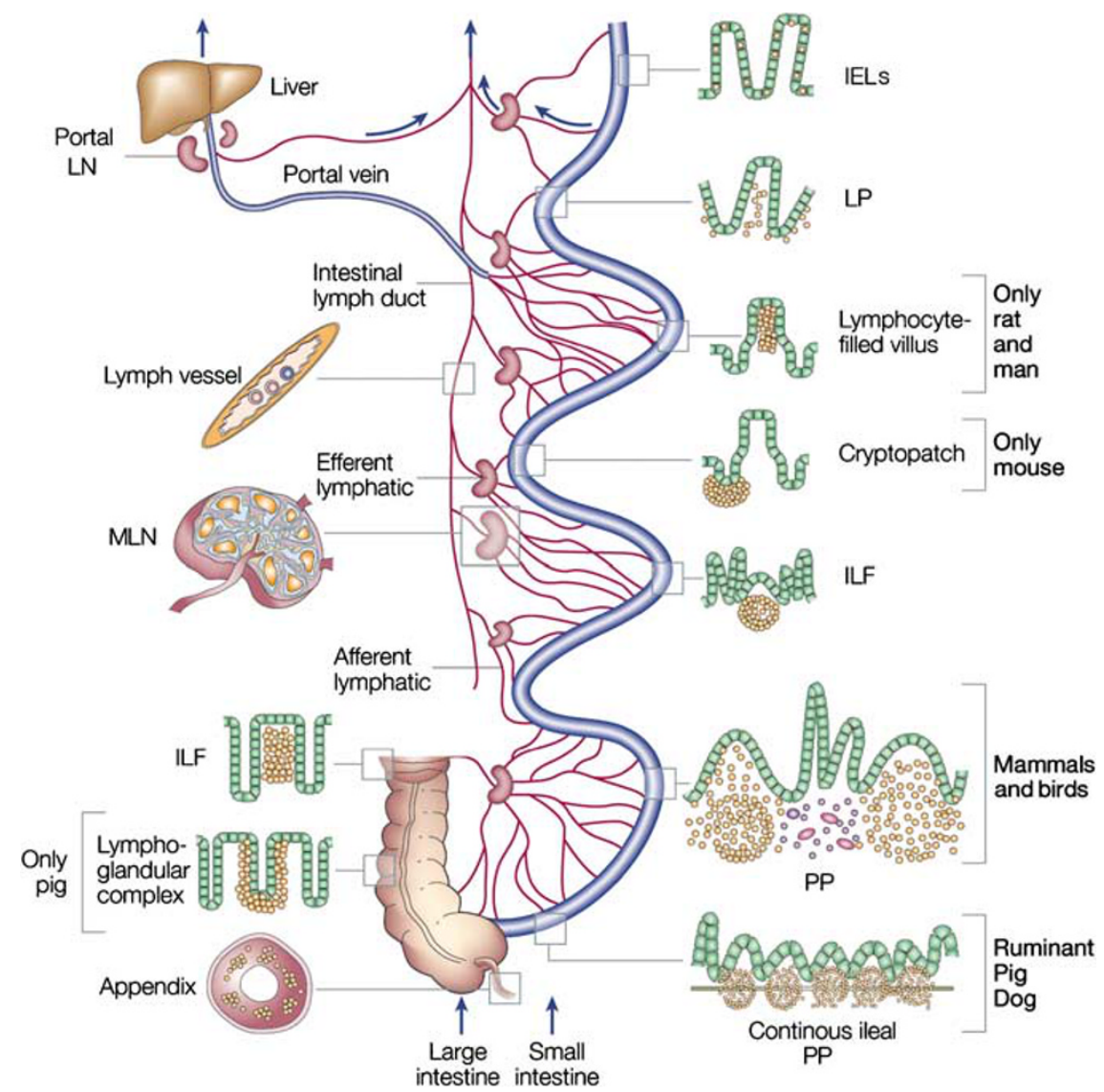

Figure 3 Delineation of lymphoid-cell distribution in various compartments of the gut wall with some species differences indicated. Lymphocytes can leave the gut wall via draining lymphatics afferent to mesenteric lymph nodes (MLNs), or via portal blood reaching the liver where important regulation of immunity apparently takes place, particularly induction of tolerance. Commonly used abbreviations (see Table 2) are shown for various aggregates of lymphoid cells such as Peyer's patches (PPs). The frequency of M cells (not indicated see Fig. 1) in the follicle-associated epithelium of PPs is highly variable among different species, and a reliable marker for these specialized epithelial cells has not been identified in humans. Note also that, in contrast to the antigen-dependent priming of B cells that takes place in PPs of mammals and birds, the continuous ileal PP present in ruminants, pigs, and dogs appears to be a primary lymphoid organ responsible for antigen-independent B-cell development, similar to the bursa of Fabricius in chicken (not indicated). This PP can be up to $2 \mathrm{~m}$ long and constitute $80-90 \%$ of the intestinal lymphoid tissue. Reptils and fish do not have PPs. In these species, the gut immune system is composed of leukocytes scattered in the epithelium and occasional small non-organized lymphoid aggregates. (Modified from Brandtzaeg and Pabst ${ }^{1}$ with permission from Elsevier.)

\section{PERSISTENT CONFUSION ABOUTTHE MALT CONCEPT}

Although GALT is the largest and the best-defined part of MALT, additional potentially inductive sites for mucosal B cells are regularly associated with the oro- and nasopharynx, such as the tonsils in humans. Interestingly, the cellular-molecular mechanisms involved in the organogenesis of various MALT structures show both similarities and still unexplained quite intriguing differences. ${ }^{1,35-39}$

Rodents lack tonsils but paired MALT structures occur on both sides of the nasopharyngeal duct dorsal to the cartilagous soft palate. ${ }^{40}$ These structures were originally referred to as NALT or "nasal-associated lymphoid tissue." 40 Grammatically, this is a misnomer, and some authors have therefore changed it to "nose-associated lymphoid tissue." We recommend, instead, "nasopharynx-associated lymphoid tissue," which in humans comprises the unpaired nasopharyngeal tonsil (also called "adenoids"-note, in plural), the paired palatine tonsils, and other smaller lymphoid structures of Waldeyer's pharyngeal ring. ${ }^{41,42}$
As mentioned above, the functional distinction between inductive and effector sites is not absolute, ${ }^{9}$ but the cues for extravasation, migration, and accumulation of naive vs. memory/ effector $\mathrm{B}$ and $\mathrm{T}$ cells within these immune compartments are nevertheless different. ${ }^{7,38,43,44}$ It is therefore confusing when authors even in major journals and textbooks refer to mucosal effector compartments and their cells (e.g., LP lymphocytes and IELs) as part of MALT. This is in direct conflict with the classical definition of a lymphoid tissue as stated in authoritative texts such as Terminologia Anatomica (International Anatomical Terminology). ${ }^{45}$

Gray's Anatomy - in an attempt at clarification-further confuses the issue; ${ }^{46}$ this text introduces the term "organized MALT" (O-MALT) for the proper MALT structures, while "diffuse MALT" (D-MALT) refers to "the disseminated population of lymphocytes within the LP and epithelial base." Although we are aware of the fact that some mucosal immunologists have used these terms, we strongly recommend that they be abandoned. Moreover, we discourage referring to the "common" 
mucosal immune system - a term that originally was introduced to highlight the integration between various inductive sites and separate effector sites. ${ }^{11}$ Although this notion has been operationally quite useful, the term now confuses this issue because recent work has revealed striking compartmentalization in the homing cues for memory/effector cells migrating to different mucosal tissues. ${ }^{7,9,38,44}$

Finally, despite the fact that mucosal immune responses are amplified in mucosa-draining local/regional LNs, they should not be included in the MALT concept. Thus, although being part of the intestinal immune system, MLNs should not be called GALT structures. This distinction is important to emphasize the fact that MALT samples antigens directly from the lumen via $\mathrm{M}$ cells. ${ }^{18}$ Likewise, although it may be appropriate to refer to head-and-neck-draining LNs as part of the mucosal immune system, we recommend to replace the acronym CONALT (for cranial-, oral-, and nasal-associated lymphoid tissue) with cervical LNs (CLNs). However, it is necessary to specify the CLNs as being deeply or superficially located because different homing mechanisms have been identified with regard to this anatomical distinction in rodents. ${ }^{47,48}$ In humans, deep cervical LNs are obtained by the classical Daniel's biopsy.

\section{CONCLUSIONS}

Several other potential MALT structures have been named in the literature, but they are not as well characterized as those mentioned in Table 2. Some examples are SALT/DALT (salivary-gland- or duct-associated lymphoid tissue), CALT (conjunctiva-associated lymphoid tissue), LDALT (lachrymaldrainage-associated lymphoid tissue), TALT (Eustachian tubeassociated lymphoid tissue), and LALT (larynx-associated lymphoid tissue).

It is strongly recommended that immunologists standardize their terminology. To draw a correct functional map of immune compartments, it requires knowledge of the actual "landscape" in terms of anatomy/histology. It is important to know the definition of a lymphoid tissue, which for mucosal compartments appropriately applies only to the organized MALT structures. The mucosal effector sites, represented by LP and surface epithelium, exert distinct functions and cannot, by established classical definition, be called lymphoid tissues; they should hence not be referred to as belonging to MALT. Also notably, different cues are operating in mucosal inductive vs. mucosal effector compartments, with regard to both organogenesis and homing mechanisms. Such knowledge is mainly derived from genetically manipulated mice, and scientists should be aware of the fact that fundamental species differences exist within the mucosal immune system.

Finally, this system should not be called the "common" mucosal immune system, which may be taken erroneously to suggest uniform integration of all mucosal effector sites. We now know that the homing of primed immune cells to the various mucosal tissues is only partially integrated because the local extravasation, migration, and retention cues are largely site-specific. ${ }^{7,9,38,44}$ The nomenclature described above for the mucosal immune system is in harmony with its compartmen- talized functional specialization as well as the classical concepts of histology.

\section{DISCLOSURE}

The author declared no conflict of interest.

(c) 2008 Society for Mucosal Immunology

\section{REFERENCES}

1. Brandtzaeg, P. \& Pabst, R. Let's go mucosal: communication on slippery ground. Trends Immunol. 25, 570-577 (2004).

2. Tomasi, T.B., Tan, E.M., Solomon, A. \& Prendergast, R.A. Characteristics of an immune system common to certain external secretions. J. Exp. Med. 121, 101-124 (1965)

3. Brandtzaeg, P. Presence of $\mathrm{J}$ chain in human immunocytes containing various immunoglobulin classes. Nature 252, 418-420 (1974).

4. Brandtzaeg, P. Mucosal and glandular distribution of immunoglobulin components: differential localization of free and bound SC in secretory epithelial cells. J. Immunol. 112, 1553-1559 (1974).

5. Brandtzaeg, P. \& Prydz, H. Direct evidence for an integrated function of $\mathrm{J}$ chain and secretory component in epithelial transport of immunoglobulins. Nature 311, 71-73 (1984)

6. Brandtzaeg, P. Human secretory immunoglobulins. III. Immunochemical and physicochemical studies of secretory lgA and free secretory piece. Acta Pathol. Microbiol. Immunol. Scand. 79, 165-188 (1971).

7. Brandtzaeg, P. Induction of secretory immunity and memory at mucosal surfaces. Vaccine 25, 5467-5484 (2007).

8. Turner, M.W., Russell, M.W., Gleeson, M., Brandtzaeg, P., Ferguson, A \& Hanson, L.Å. et al. Terminology: nomenclature of immunoglobulin A and other proteins of the mucosal immune system. Bull. World Health Organ. 76, 427-428 (1998).

9. Brandtzaeg, P. \& Johansen, F.-E. Mucosal B cells: phenotypic characteristics, transcriptional regulation, and homing properties. Immunol. Rev. 206, 32-63 (2005).

10. Fagarasan, S. \& Honjo, T. Intestinal IgA synthesis: regulation of front-line body defences. Nat. Rev. Immunol. 3, 63-72 (2003).

11. McDermott, M.R. \& Bienenstock, J. Evidence for a common mucosal immunologic system. I. Migration of B immunoblasts into intestinal, respiratory, and genital tissues. J. Immunol. 122, 1892-1898 (1979).

12. Tschernig, T. \& Pabst, R. Bronchus-associated lymphoid tissue (BALT) is not present in the normal adult lung but in different diseases. Pathobiology $68,1-8$ (2000).

13. Macpherson, A.J., McCoy, K., Johansen, F.-E. \& Brandtzaeg, P. The immune geography of IgA induction and function. Mucosal Immunol., in press (2008).

14. Boursier, L., Gordon, J.N., Thiagamoorthy, S., Edgeworth, J.D. \& Spencer, J. Human intestinal IgA response is generated in the organized gut-associated lymphoid tissue but not in the lamina propria. Gastroenterology 128, 1879-1889 (2005).

15. He, B., Xu, W., Santini, P.A., Polydorides, A.D., Chiu, A. \& Estrella, J. et al. Intestinal bacteria trigger $T$ cell-independent immunoglobulin $A(2)$ class switching by inducing epithelial-cell secretion of the cytokine APRIL. Immunity 26, 812-826 (2007).

16. Kett, K., Baklien, K., Bakken, A., Kral, J.G., Fausa, O. \& Brandtzaeg, P. Intestinal B-cell isotype response in relation to local bacterial load: evidence for immunoglobulin A subclass adaptation. Gastroenterology 109, 819-825 (1995)

17. Lefrancois, L. \& Puddington, L. Intestinal and pulmonary mucosal T cells: local heroes fight to maintain the status quo. Annu. Rev. Immunol. 24, 681-704 (2006)

18. Neutra, M.R., Mantis, N.J. \& Kraehenbuhl, J.P. Collaboration of epithelial cells with organized mucosal lymphoid tissues. Nat. Immunol. 2, 1004-1009 (2001).

19. Moghaddami, M., Cummins, A. \& Mayrhofer, G. Lymphocyte-filled villi: comparison with other lymphoid aggregations in the mucosa of the human small intestine. Gastroenterology 115, 1414-1425 (1998).

20. Onai, N. et al. Pivotal role of CCL25 (TECK)-CCR9 in the formation of gut cryptopatches and consequent appearance of intraepithelial T lymphocytes. Int. Immunol. 14, 687-694 (2002). 
21. Guy-Grand, D., Azogui, O., Celli, S., Darche, S., Nussenzweig, M.C., Kourilsky, P. et al. Extrathymic T cell lymphopoiesis: ontogeny and contribution to gut intraepithelial lymphocytes in athymic and enthymic mice. J. Exp. Med. 197, 333-341 (2003).

22. Pabst, O., Herbrand, H., Friedrichsen, M., Velaga, S., Dorsch, M. Berhardt, G. et al. Adaptation of solitary intestinal lymphoid tissue in response to microbial and chemokine receptor CCR7 signaling. $J$ Immunol 177, 6824-6832 (2006).

23. Craig, S.W. \& Cebra, J.J. Peyer's patches: an enriched source of precursors for IgA-producing immunocytes in the rabbit. J. Exp. Med. 134, 188-200 (1971).

24. McWilliams, M., Phillips-Quagliata, J.M. \& Lamm, M.E. Mesenteric lymph node $B$ lymphoblasts which home to the small intestine are precommitted to lgA synthesis. J. Exp. Med. 145, 866-875 (1977).

25. Guy-Grand, D., Griscelli, C. \& Vassalli, P. The gut-associated lymphoid system: nature and properties of the large dividing cells. Eur. J. Immunol. 4, 435-443 (1974).

26. Roux, M.E., McWilliams, M., Phillips-Quagliata, J.M. \& Lamm, M.E. Differentiation pathway of Peyer's patch precursors of IgA plasma cells in the secretory immune system. Cell Immunol. 61, 141-153 (1981).

27. Parrott, D.M. The gut as a lymphoid organ. Clin. Gastroenterol. 5, 211-228 (1976)

28. Lamm, M.E. Cellular aspects of immunoglobulin A. Adv. Immunol. 22, 223-290 (1976).

29. Dunn-Walters, D.K., Isaacson, P.G. \& Spencer, J. Sequence analysis of human IgVH genes indicates that ileal lamina propria plasma cells are derived from Peyer's patches. Eur. J. Immunol. 27, 463-467 (1997).

30. Cornes, J.S. Number, size and distribution of Peyer's patches in the human small intestine. Gut 6, 225-233 (1965).

31. Spencer, J. \& MacDonald, T.T. Ontogeny of human mucosal immunity. In Ontogeny of the Immune System of the Gut (MacDonald, T.T., ed) 23-50 (CRC Press, Boca Raton, FL, 1990).

32. Trepel, F. Number and distribution of lymphocytes in man. A critical analysis. Klin. Wochenschr. 52, 511-515 (1974).

33. O'Leary, A.D. \& Sweeney, E.C. Lymphoglandular complexes of the colon: structure and distribution. Histopathology 10, 267-283 (1986).

34. Hamada, H., Hiroi, T., Nishiyama, Y., Takahashi, H., Masunaga, Y., Hachimura, S et al. Identification of multiple isolated lymphoid follicles on the antimesenteric wall of the mouse small intestine. J. Immunol. 168, 57-64 (2002).

35. Dohi, T., Rennert, P.D., Fujihashi, K., Kiyono, H., Shirai, Y., Kawamura, Y.I. et al. Elimination of colonic patches with lymphotoxsin $\beta$ receptor-lg prevents Th2 cell-type colitis. J. Immunol. 167, 2781-2790 (2001).
36. Carlsen, H.S., Baekkevold, E.S., Johansen, F.E., Haraldsen, G., Brandtzaeg, P. B cell attracting chemokine 1 (CXCL13) and its receptor CXCR5 are expressed in normal and aberrant gut associated lymphoid tissue. Gut 51, 364-371 (2002).

37. Harmsen, A., Kusser, K., Harson, L., Tighe, M., Sunshine, M.J., Sedgwick, J.D. et al. Cutting edge: organogenesis of nasal-associated lymphoid tissue (NALT) occurs independently of lymphotoxin- $\alpha(L T \alpha)$ and retinoic acid receptor-related orphan receptor- $\gamma$, but the organization of NALT is LT $\alpha$ dependent. J. Immunol. 168, 986-990 (2002).

38. Kiyono, H. \& Fukuyama, S. NALT- versus Peyer's-patch-mediated mucosal immunity. Nat. Rev. Immunol. 4, 699-710 (2004).

39. Fukuyama, S., Nagatake, T., Kim, D.Y., Takamura, K., Park, E.J. \& Kaisho, T. et al. Cutting edge: uniqueness of lymphoid chemokine requirement for the initiation and maturation of nasopharynx-associated lymphoid tissue organogenesis. J. Immunol. 177, 4276-4280 (2006).

40. Kuper, C.F., Koornstra, PJ, Hameleers, DM, Biewenga, J, Spit, BJ \& Duijvestijn, AM et al. The role of nasopharyngeal lymphoid tissue. Immunol. Today 13, 219-224 (1992).

41. Perry, M. \& Whyte, A. Immunology of the tonsils. Immunol. Today 19 , 414-421 (1998)

42. Brandtzaeg, P. Immunology of tonsils and adenoids: everything the ENT surgeon needs to know. Int. Congr. Ser. (ICS) 1254, 89-99 (2003) (Elsevier)/Int. J. Pediatr. Otorhinolaryngol, 2003, 67 (Suppl 1): S69-S76.

43. Kunkel, E.J. \& Butcher, E.C. Chemokines and the tissue-specific migration of lymphocytes. Immunity 16, 1-4 (2002).

44. Johansen, F.-E., Baekkevold, E.S., Carlsen, H.S., Farstad, I.N., Soler, D. \& Brandtzaeg, P. Regional induction of adhesion molecules and chemokine receptors explains disparate homing of human $B$ cells to systemic and mucosal effector sites: dispersion from tonsils. Blood 106, 593-600 (2005).

45. Federative Committee on Anatomical Terminology (FCAT). Terminologia Anatomica; International Anatomical Terminology 52, 100-101Thieme Stuttgart, New York, 1998).

46. Williams, P.L. (ed) Gray's Anatomy 38th edn, 144-145 (Churchill Livingstone, Edinburgh, 1995).

47. Csencsits, K.L., Jutila, M.A. \& Pascual, D.W. Mucosal addressin expression and binding-interactions with naive lymphocytes vary among the cranial, oral, and nasal-associated lymphoid tissues. Eur. J. Immunol. 32, 3029-3039 (2002).

48. Csencsits, K.L. \& Pascual, D.W. Absence of L-selectin delays mucosal B cell responses in nonintestinal effector tissues. J. Immunol. 169, 5649-5659 (2002). 